\title{
Gradient for the acoustic VTI full waveform inversion based on the instantaneous traveltime sensitiv- ity kernels
}

Ramzi Djebbi* and Tariq Alkhalifah, King Abdullah University of Science and Technology

\section{SUMMARY}

The instantaneous traveltime is able to reduce the non-linearity of full waveform inversion (FWI) that originates from the wrapping of the phase. However, the adjoint state method in this case requires a total of 5 modeling calculations to compute the gradient. Also, considering the larger modeling cost for anisotropic wavefield extrapolation and the necessity to use a line-search algorithm to estimate a step length that depends on the parameters scale, we propose to calculate the gradient based on the instantaneous traveltime sensitivity kernels. We, specifically, use the sensitivity kernels computed using dynamic ray-tracing to build the gradient. The resulting update is computed using a matrix decomposition and accordingly the computational cost is reduced. We consider a simple example where an anomaly is embedded into a constant background medium and we compute the update for the VTI wave equation parameterized using $v_{h}, \eta$ and $\varepsilon$.

\section{INTRODUCTION}

Full waveform inversion (FWI) aims to invert the full recorded data content to recover an accurate Earth model (Virieux and Operto, 2009). Recently, the improvements in seismic data acquisition, with large offsets and broad frequency content, as well as the availability of the advanced computational devices, paved the way for FWI to be more practical (Pratt et al., 1996; Shin and Min, 2006; Choi and Alkhalifah, 2013). In fact, FWI still suffers from the high nonlinearity of the objective function, which may result in convergence to local minima (Virieux and Operto, 2009). Woodward (1992) introduced wave equation tomography as a reduced form of FWI, where the misfit is the difference between a finite-frequency traveltime of the observed and synthetic data. Choi and Alkhalifah (2013) introduced the instantaneous traveltime as an inversion observable based on a derivative operator applied to the wavefield. The derivative operator associated with a variable damping factor is able to unwrap the phase as well as acting as a tomographic inversion and an FWI one with a single objective function (Alkhalifah and Choi, 2014).

An additional issue appears when we start to invert for more than one parameter. The ambiguity or trade-off between the different parameters (Plessix and Cao, 2011; Gholami et al., 2013; Alkhalifah and Plessix, 2014; Métivier et al., 2014) affects the accuracy of the inverted model. Métivier et al. (2014) showed that the Hessian matrix is crucial to correct for the cross-talk and scale difference between the parameter classes. For transversely isotropic media with vertical axis of symmetry (VTI media), Gholami et al. (2013) and Alkhalifah and Plessix (2014) analyzed the trade-off based on the radiation patterns. Djebbi et al. (2015) derived the sensitivity kernels for anisotropy parameter perturbation and analyzed the trade- off along the wave-path. For conventional surface recorded data, where diving waves (transmission) and reflections are available, a parameterization of the wave equation using the horizontal velocity $v_{h}$, and the anisotropy parameters $\eta$ and $\varepsilon$ shows the least trade-off (Alkhalifah and Plessix, 2014).

Conventionally, FWI is implemented using the adjoint state method (Tarantola, 1984) to compute the gradient. Liu et al. (2014) proposed to use the Born sensitivity kernels (Woodward, 1992; Dahlen et al., 2000) in isotropic media to compute the gradient by matrix decomposition. Each Born kernel requires two modeling steps: one from the source location and another from the receiver location. The authors showed that the method is efficient when the number of sources is more than half of the number of receivers. The inversion method had been applied successfully in the context of global seismology inversions where this condition holds (Dahlen et al., 2000). In this abstract, we propose to invert the instantaneous traveltime (Choi and Alkhalifah, 2013; Alkhalifah and Choi, 2014) for VTI media. The wave equation (Duveneck et al., 2008) is parameterized using $v_{h}, \eta$ and $\varepsilon$ and we compute the update direction using the sensitivity kernels. As the model is usually updated from low to high wavenumbers (relying on smooth models in the begining), we use dynamic ray tracing (Červenỳ, 2005) as an effective alternative to wavefield modeling to compute the instantaneous traveltime kernels (Djebbi and Alkhalifah, 2014) for the first few iterations of the inversion. Added to the superior computational efficiency, dynamic ray-tracing provides the ray direction information necessary to handle the scattering angles dependency of the anisotropic sensitivity kernels. The matrix decomposition method (Liu et al., 2014) is used to avoid the need to store the Fréchet derivative matrix.

\section{THEORY}

The instantaneous traveltime is introduced as a finite frequency measure of traveltime. Choi and Alkhalifah (2013) showed that the instantaneous traveltime associated with a damping factor unwraps the phase and reduces the non-linearity of the inversion. The instantaneous traveltime is given as,

$$
\tau(\omega)=\operatorname{Im}\left(\frac{\frac{\partial \hat{u}_{j}(\omega)}{\partial \omega}}{\hat{u}_{j}(\omega)}\right),
$$

where Im is the imaginary part symbol and $\hat{u}_{j}(\omega)$ is the frequency domain wavefield at receiver $j$.

When a high exponential damping is applied to the wavefield, the instantaneous traveltime is equivalent to the first arrival traveltime and the inversion is considered as a traveltime tomography. As we decrease the damping factor, the estimated traveltime includes more information about later events (reflections) and the model update will contain high wavenumbers necessary to invert for high resolution models. The objec- 


\section{Gradient for the acoustic VTI full waveform inversion}

tive function using the instantaneous traveltime is expressed for a single shot and a single frequency as,

$$
E=\sum_{j=1}^{n r} \frac{1}{2}\left[\operatorname{Im}\left(\frac{\frac{\partial \hat{u}_{j}(\omega)}{\partial \omega}}{\hat{u}_{j}(\omega)}\right)-\operatorname{Im}\left(\frac{\frac{\partial \hat{d}_{j}(\omega)}{\partial \omega}}{\hat{d}_{j}(\omega)}\right)\right]^{2},
$$

where $\hat{u}_{j}$ is the modeled wavefield, $\hat{d}_{j}$ is the observed wavefield at the receiver $j$ and $n r$ is the number of receivers.

Considering the Helmholtz equation in a matrix form:

$$
\mathbf{S}(\omega) \hat{\mathbf{u}}(\omega)=\mathbf{f},
$$

where $\mathbf{S}$ is the impedence matrix, $\hat{\mathbf{u}}$ is the wavefield and $\mathbf{f}$ the source function, Choi and Alkhalifah (2013) expressed the gradient of the objective function as,

$$
\begin{aligned}
\frac{\partial E}{\partial m_{i}} & =\operatorname{Im}\left[\left(-\frac{\partial \mathbf{S}}{\partial m_{i}} \frac{\partial \hat{\mathbf{u}}}{\partial \omega}-\frac{\partial^{2} \mathbf{S}}{\partial m_{i} \partial \omega} \hat{\mathbf{u}}\right)^{T} \mathbf{S}^{-1} \mathbf{r}_{1}+\left(-\frac{\partial \mathbf{S}}{\partial m_{i}} \hat{\mathbf{u}}\right)^{T}\right. \\
& \left.\times \mathbf{S}^{-1}\left\{\left(-\frac{\partial \mathbf{S}}{\partial \omega}\right)^{T} \mathbf{S}^{-1} \mathbf{r}_{1}\right\}\right]-\operatorname{Im}\left[\left(-\frac{\partial \mathbf{S}}{\partial m_{i}} \hat{\mathbf{u}}\right)^{T} \mathbf{S}^{-1} \mathbf{r}_{2}\right],
\end{aligned}
$$

$m_{i}$ is one model parameter. The elements of the residual vectors $\mathbf{r}_{1}$ and $\mathbf{r}_{2}$ are given respectively as:

$$
\begin{gathered}
r_{1 j}=\frac{1}{\hat{u}_{j}} \operatorname{Im}\left[\left(\frac{\partial \hat{u}_{j}}{\partial \omega}\right) / \hat{u}_{j}-\left(\frac{\partial \hat{d}_{j}}{\partial \omega}\right) / \hat{d}_{j}\right] \\
r_{2 j}=\frac{\left(\partial \hat{u}_{j} / \partial \omega\right)}{\hat{u}_{j}^{2}} \operatorname{Im}\left[\left(\frac{\partial \hat{u}_{j}}{\partial \omega}\right) / \hat{u}_{j}-\left(\frac{\partial \hat{d}_{j}}{\partial \omega}\right) / \hat{d}_{j}\right]
\end{gathered}
$$

To compute the gradient, a total of 5 modeling steps is needed: two forward modeling operations to compute $\hat{\mathbf{u}}$ and $\partial \hat{\mathbf{u}} / \partial \omega$, and 3 adjoint state back-propagations of $\mathbf{r}_{1}, \mathbf{r}_{2}$, and $\left(-\frac{\partial \mathbf{S}}{\partial \omega}\right)^{T} \mathbf{S}^{-1} \mathbf{r}_{1}$

Although the modeling operator $\mathbf{S}$ is factorized once per iteration and used for all the modeling steps, the computational cost is still relatively high, especially if a line search algorithm is used to optimize the step length. Also, for VTI media, the modeling computational cost is large compared to the isotropic case.

To take advantage of the instantaneous traveltime approach in solving the phase wrapping problem and to reduce the computational cost, we propose to use Liu et al. (2014) approach where the gradient is computed based on Born kernels and using a matrix decomposition of the Fréchet derivative.

\section{The traveltime sensitivity kernels}

The sensitivity kernels or the Fréchet derivatives relate a perturbation in the recorded data to a perturbation in the Earth model. The derivation of the instantaneous traveltime sensitivity kernels is composed of two main steps: First, we derive the Born approximation that relates the wavefield perturbation to a perturbation in the anisotropy parameters. Then, we extract the traveltime perturbation using the instantaneous traveltime (Djebbi and Alkhalifah, 2014)
We start with the VTI system of equations (Duveneck et al., 2008 ) and we combine the two equations into a single $4^{\text {th }}$ order equation. The model is parameterized using the horizontal velocity $v_{h}, \eta$ and $\varepsilon$ parameters. Perturbing in the three Earth model parameters results in a wavefield perturbation of the form:

$$
\begin{aligned}
\mathrm{d} u\left(\mathbf{x}_{\mathbf{s}}, \mathbf{x}_{\mathbf{r}}, \mathbf{x}, \omega\right)=\int & {\left[K_{B}^{\left(v_{h}\right)}\left(\mathbf{x}_{\mathbf{r}}, \mathbf{x}_{\mathbf{s}}, \mathbf{x}, \omega\right) \mathrm{d} v_{h}+K_{B}^{(\eta)}\left(\mathbf{x}_{\mathbf{r}}, \mathbf{x}_{\mathbf{s}}, \mathbf{x}, \omega\right) \mathrm{d} \eta\right.} \\
& \left.+K_{B}^{(\varepsilon)}\left(\mathbf{x}_{\mathbf{r}}, \mathbf{x}_{\mathbf{s}}, \mathbf{x}, \omega\right) \mathrm{d} \varepsilon\right] \mathrm{d} \mathbf{x},
\end{aligned}
$$

where $K_{B}^{\left(v_{h}\right)}, K_{B}^{(\eta)}$ and $K_{B}^{(\varepsilon)}$ are the wavefield sensitivity kernels or Born kernels.

For a 2-D VTI medium, the Born kernels are given as (Djebbi et al., 2015),

$$
\begin{aligned}
K_{B}{ }^{\left(v_{h}\right)}\left(\mathbf{x}_{\mathbf{r}}, \mathbf{x}_{\mathbf{s}}, \mathbf{x}, \omega\right) & =-\frac{\omega^{2}}{v_{0}^{4}} u_{0}\left(\mathbf{x}, \mathbf{x}_{\mathbf{s}}, \omega\right) G_{0}\left(\mathbf{x}, \mathbf{x}_{\mathbf{r}}, \omega\right) \\
K_{B}{ }^{(\eta)}\left(\mathbf{x}_{\mathbf{r}}, \mathbf{x}_{\mathbf{s}}, \mathbf{x}, \omega\right) & =-\left(u_{0}\left(\mathbf{x}, \mathbf{x}_{\mathbf{s}}, \omega\right) \partial_{z z} G_{0}\left(\mathbf{x}, \mathbf{x}_{\mathbf{r}}, \omega\right)\right. \\
& \left.+\partial_{z z} u_{0}\left(\mathbf{x}, \mathbf{x}_{\mathbf{s}}, \omega\right) G_{0}\left(\mathbf{x}, \mathbf{x}_{\mathbf{r}}, \omega\right)\right) \\
& -\frac{2 v_{0}^{2}}{\omega^{2}} \partial_{z z} u_{0}\left(\mathbf{x}, \mathbf{x}_{\mathbf{s}}, \omega\right) \partial_{z z} G_{0}\left(\mathbf{x}, \mathbf{x}_{\mathbf{r}}, \omega\right) \\
K_{B}{ }^{(\varepsilon)}\left(\mathbf{x}_{\mathbf{r}}, \mathbf{x}_{\mathbf{s}}, \mathbf{x}, \omega\right) & =-\left(u_{0}\left(\mathbf{x}, \mathbf{x}_{\mathbf{s}}, \omega\right) \partial_{z z} G_{0}\left(\mathbf{x}, \mathbf{x}_{\mathbf{r}}, \omega\right)\right. \\
& \left.+\partial_{z z} u_{0}\left(\mathbf{x}, \mathbf{x}_{\mathbf{s}}, \omega\right) G_{0}\left(\mathbf{x}, \mathbf{x}_{\mathbf{r}}, \omega\right)\right)
\end{aligned}
$$

where $u_{0}\left(\mathbf{x}, \mathbf{x}_{\mathbf{s}}, \omega\right)$ and $G_{0}\left(\mathbf{x}, \mathbf{x}_{\mathbf{r}}, \omega\right)$ are the source wavefield and the receiver Green's function, respectively.

The instantaneous traveltime kernels are obtained as,

$$
K^{(p)}\left(\mathbf{x}_{\mathbf{r}}, \mathbf{x}_{\mathbf{s}}, \mathbf{x}, \omega\right)=\operatorname{Im}\left(\frac{\partial K_{B}^{(p)}\left(\mathbf{x}_{\mathbf{r}}, \mathbf{x}_{\mathbf{s}}, \mathbf{x}, \omega\right) / \partial \omega}{G_{0}\left(\mathbf{x}_{\mathbf{s}}, \mathbf{x}_{\mathbf{r}}, \omega\right)}\right),
$$

where $p=\left(v_{h}, \eta, \varepsilon\right)$. Djebbi and Alkhalifah (2014) showed that the instantaneous traveltime kernels are more general and form the unwrapped phase version of the Rytov approximation for the phase.

\section{Gradient calculation using the instantaneous traveltime kernels}

The instantaneous traveltime kernel given in equation (9) is the single frequency kernel for a specific source and receiver pair and describes the sensitivity at a specific subsurface point located at $\mathbf{x}$. We define the number of the subsurface model points as $n=n_{p} \times n_{x} \times n_{z}$, where $n_{p}$ is the number of parameters ( $n_{p}=3$ for VTI media), and $n_{x}$ and $n_{z}$ the model dimensions. Also, we define the number of all possible combinations of sources and receivers (data size) as $m$. The total size of the Fréchet derivative matrix is $(n \times m)$. In order to use the Fréchet derivative matrix explicitly, Liu et al. (2014) used a matrix decomposition to reduce the large memory requirement needed to store the matrix.

The gradient of the instantaneous traveltime objective function (2) based on the sensitivity kernels is given as:

$$
\frac{\partial E}{\partial \mathbf{m}}=-\mathbf{K}^{T} \Delta \tau(\omega)=-\mathbf{K}^{T} \operatorname{Im}\left[\left(\frac{\partial \hat{\mathbf{u}}}{\partial \omega}\right) / \hat{\mathbf{u}}-\left(\frac{\partial \hat{\mathbf{d}}}{\partial \omega}\right) / \hat{\mathbf{d}}\right]
$$

where $\Delta \tau(\omega)$ is the instantaneous traveltime residual and $\mathbf{K}$ the sensitivity kernel matrix. 


\section{Gradient for the acoustic VTI full waveform inversion}

Based on the matrix decomposition method (Liu et al., 2014), the gradient is computed without saving the sensitivity kernels matrix $\mathbf{K}$ as:

$$
\frac{\partial E}{\partial \mathbf{m}}=\left(\begin{array}{c}
k_{11} \\
\vdots \\
k_{1 j} \\
\vdots \\
k_{1 n}
\end{array}\right) \Delta \tau_{1}+\cdots+\left(\begin{array}{c}
k_{i 1} \\
\vdots \\
k_{i j} \\
\vdots \\
k_{i n}
\end{array}\right) \Delta \tau_{i}+\cdots+\left(\begin{array}{c}
k_{m 1} \\
\vdots \\
k_{m j} \\
\vdots \\
k_{m n}
\end{array}\right) \Delta \tau_{m}(11)
$$

where $k_{i j}$ is the sensitivity kernel given by equation (9) for a model parameter point " $i$ " and source/receiver pair " $j$ ".

Thus, the proposed approach is to compute the data residual using conventional wavefield modeling and use dynamic raytracing to estimate the instantaneous traveltime sensitivity kernels. The resulting gradient will benefit from the ray-tracing efficiency in computing the kernels. For anisotropy, as shown by equation (8) the kernels are angular dependent, hence dynamic ray-tracing handles this dependency explicitly using the calculated ray-parameter information.

\section{NUMERICAL EXAMPLE}

We consider the case of an anomaly embedded into a homogeneous background medium. The properties of the background medium are: $v_{h}=2000 \mathrm{~ms}^{-1}, \eta=0.1$ and $\varepsilon=0.2$. The anomaly perturbation is $+25 \%$ with respect to the horizontal velocity and $+50 \%$ for $\eta$ and $\varepsilon$. The circular anomaly is located at the center of the model $\left(x_{a}=2500 \mathrm{~m}, z_{a}=2500 \mathrm{~m}\right)$, as shown in Figure 1. Here, we consider a transmission experiment as usually done in the early iterations of FWI, and thus, we aim to match the diving waves (the kinematics of the wavefield). Figure 1 shows also the sources line (51 sources) located at $x=50 \mathrm{~m}$ and the receivers line (251 receivers) located at $x=4950 \mathrm{~m}$.

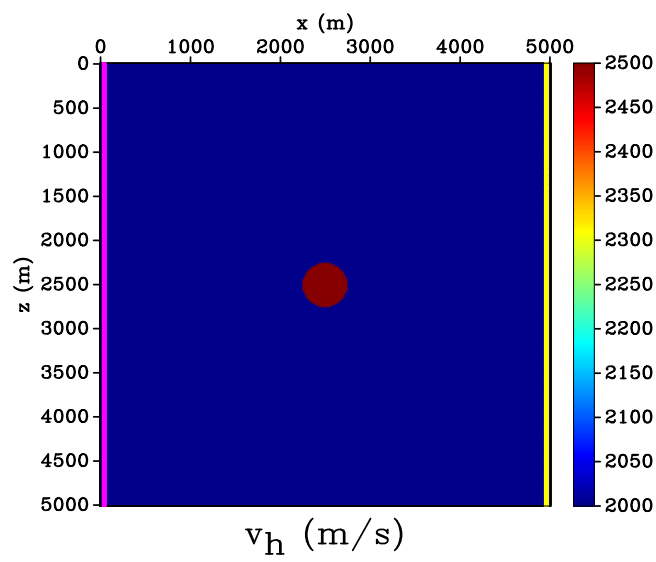

Figure 1: Horizontal velocity model with the anomaly. The anomaly is $+25 \%$ of the background constant velocity. The sources are located along the vertical purple line at $x=50 \mathrm{~m}$. The receivers along the vertical yellow line at $x=4950 \mathrm{~m}$.

The first step to compute the gradient is to estimate the instantaneous traveltime residual vector $\Delta \tau(\omega)$. We model the

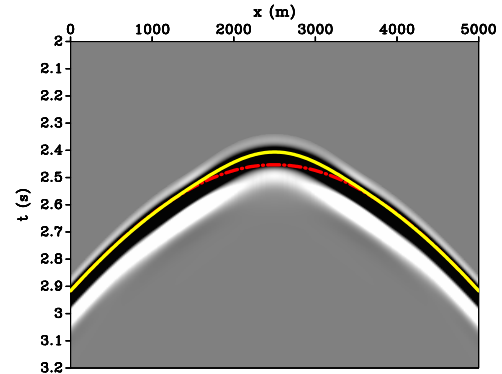

(a)

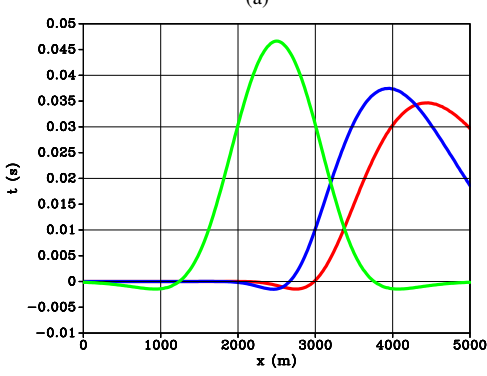

(b)

Figure 2: Estimated instantaneous traveltime for frequency $f=1 \mathrm{~Hz}$ and damping coefficient $\alpha=25 \mathrm{~s}^{-1}$. (a) The estimated instantaneous traveltime on the background model shown in red, and on the model with anomaly shown in yellow, overlaid to the damped shot gather. (b) Estimated residuals for different shots, red: shot at $z_{s}=500 \mathrm{~m}$, blue: shot at $z_{s}=1000$ $\mathrm{m}$ and green: shot at $z_{s}=2500 \mathrm{~m}$.

data using a finite difference acoustic VTI wave extrapolation. We use a Ricker wavelet with a peak frequency of $5 \mathrm{~Hz}$ as a source function. The obtained data is damped using an exponential damping function $d=e^{-\alpha t}$. In conventional acquisition geometry, large damping factors enhance the early arrivals and a good strategy is to start with high damping then relax it throughout the inversion to include later events and achieve high resolution inverted models (Choi and Alkhalifah, 2013). Figure 2 shows an example of the estimated instantaneous traveltime and the residuals. The frequency is $1 \mathrm{~Hz}$ and the damping factor is $\alpha=25 \mathrm{~s}^{-1}$. Figure 2a shows a damped shot gather and the estimated instantaneous traveltime in the background model (red curve) and in the model with anomaly (yellow curve) for a shot at $z_{s}=2500 \mathrm{~m}$. Figure $2 \mathrm{~b}$ shows the estimated residuals for different shots. The effect of the anomaly is clearly observed in the residuals.

The sensitivity kernels for the instantaneous traveltime are computed using equation (9). We need to estimate the Green's functions excited at the source and receiver locations. As a single kernel requires two modeling steps, the approach based on full Green's functions modeling is only effective when the number of sources is more than half the number of receivers. Here, we use dynamic ray-tracing to estimate the Green's functions and their derivatives. The dynamic ray-tracing approach is based on a Runge-Kutta method and provides a map of the traveltime, amplitude and ray direction on the grid for each shot (from source/receiver location). Therefore, the Green's 


\section{Gradient for the acoustic VTI full waveform inversion}

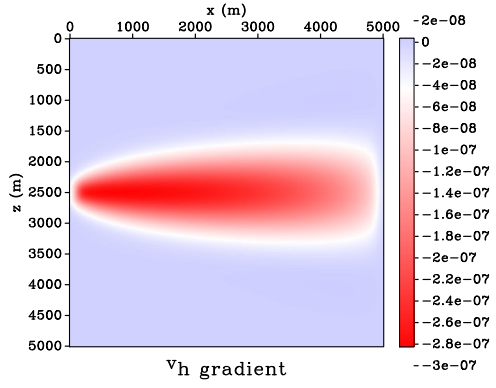

(a)

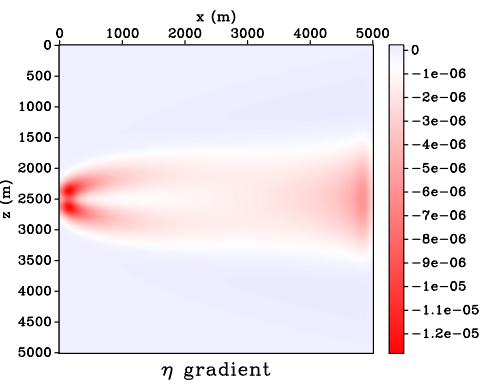

(b)

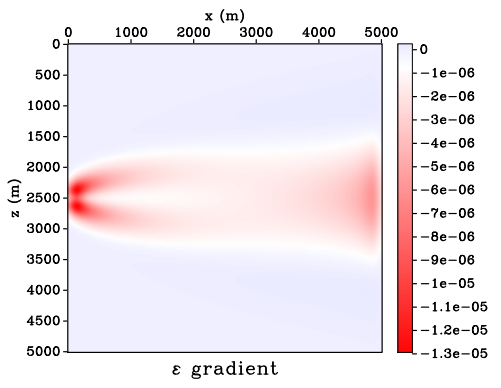

(c)

Figure 3: Gradient for a single shot located at $z_{s}=2500 \mathrm{~m}$. (a) Horizontal velocity $v_{h}$, (b) $\eta$ parameter and (c) $\varepsilon$ parameter.

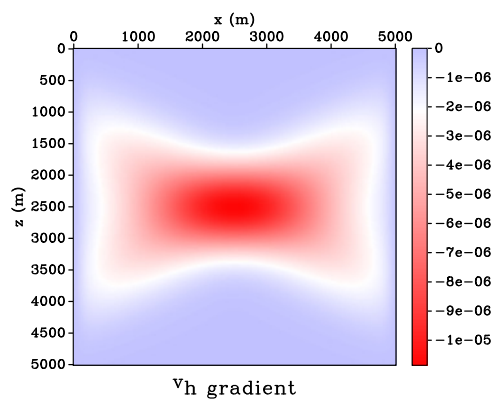

(a)

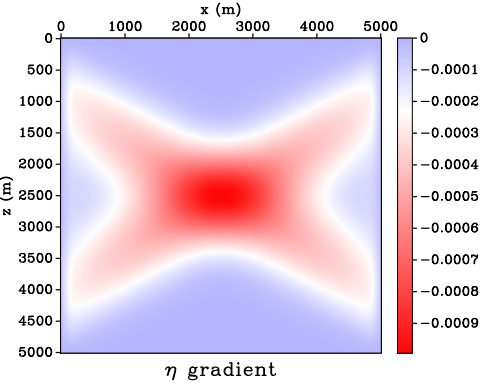

(b)

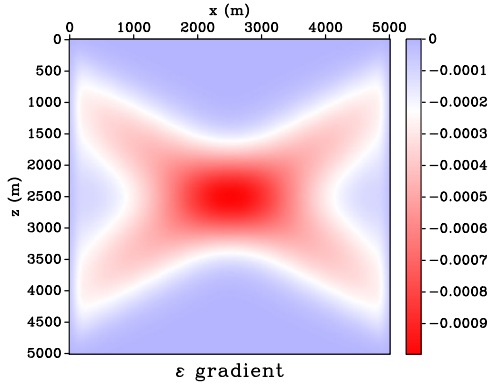

(c)

Figure 4: Gradient for all 51 shots along the vertical line of sources. (a) Horizontal velocity $v_{h}$, (b) $\eta$ parameter and (c) $\varepsilon$ parameter.

functions derivatives are easily computed and the anisotropy parameter directional radiation pattern is handled. A simple multiplication of each kernel with the instantaneous traveltime residual is then performed to obtain the gradient.

Figure 3 shows the gradient for a single shot located at $z_{s}=$ $2500 \mathrm{~m}$. The angular dependency of the anisotropy parameters (Alkhalifah and Plessix, 2014; Djebbi et al., 2015) is observed in this simple example. The horizontal velocity behavior in $\left(v_{h}, \eta, \varepsilon\right)$ parameterization of the wave equation is isotropic, thus the gradient here does not depend on the angle between the source and receiver. On the other hand, the $\eta$ and $\varepsilon$ radiation patterns show that $\eta$ maximum sensitivity is at $45^{\circ}$ scattering angles, and $\varepsilon$ maximum sensitivity is for $90^{\circ}$ scattering angles (Alkhalifah and Plessix, 2014). Using all the shots, the final gradient (Figure 4) shows a maximum update in the center of the model, which is the location of the anomaly. Then, the gradient can be used to invert for the anisotropic parameters. The damping factor is relaxed with advancing the iterations and also we increase the treated frequency to recover a high resolution model. All will be shown at the meeting.

\section{DISCUSSION AND CONCLUSIONS}

The instantaneous traveltime is able to avoid the wrapping phenomena observed during inversion problems. The gradient computation using the adjoint state method for the instantaneous traveltime objective function requires 5 total modeling steps, which makes the method computationally expensive. Accordingly, the use of the sensitivity kernels to compute the gradients for the VTI model parameters inversion is a good alternative as only one modeling is needed to compute the residuals. The kernels are computed using dynamic ray tracing, which reduces the computational cost and also provides the direction information needed to handle the anisotropy parameters angular dependency.

The method is applied to compute the gradient for a transmission geometry for a VTI wave equation parameterized using $\left(v_{h}, \eta, \varepsilon\right)$. For a single shot, the gradients show the angular effects for $\eta$ and $\delta$ parameters. However, although the parameterization is the best suited for a data dominated with transmissions, a trade-off is observed when considering all the shots. In fact, if more than one shot is considered, a wide range of scattering angles are included and thus the data become sensitive to all the parameters. The example shows that even for a simple anomaly model, to decide on the step length is a complicated task. Scaling and trade-off between the parameters should be taken into account by the incorporation of the Hessian matrix.

\section{ACKNOWLEDGEMENTS}

We would like to thank King Abdullah University of Science and Technology for the financial support. We thank Yunseok Choi and Nabil Masmoudi for the useful suggestions. 


\section{EDITED REFERENCES}

Note: This reference list is a copyedited version of the reference list submitted by the author. Reference lists for the 2015 SEG Technical Program Expanded Abstracts have been copyedited so that references provided with the online metadata for each paper will achieve a high degree of linking to cited sources that appear on the Web.

\section{REFERENCES}

Alkhalifah, T., and Y. Choi, 2014, From tomography to full-waveform inversion with a single objective function: Geophysics, 79, no. 2, R55-R61. http://dx.doi.org/10.1190/geo2013-0291.1.

Alkhalifah, T., and R.-E. Plessix, 2014, A recipe for practical full-waveform inversion in anisotropic media: An analytical parameter resolution study: Geophysics, 79, no. 3, R91R101. http://dx.doi.org/10.1190/geo2013-0366.1.

Cerveny, V., 2005, Seismic ray theory: Cambridge University Press.

Choi, Y., and T. Alkhalifah, 2013, Frequency-domain waveform inversion using the phase derivative: Geophysical Journal International, 195, no. 3, 1904-1916. http://dx.doi.org/10.1093/gji/ggt351.

Dahlen, F. A., S.-H. Hung, and G. Nolet, 2000, Frechet kernels for finite-frequency traveltimes - I: theory: Geophysical Journal International, 141, no. 1, 157-174. http://dx.doi.org/10.1046/j.1365246X.2000.00070.X.

Djebbi, R., and T. Alkhalifah, 2014, Traveltime sensitivity kernels for wave equation tomography using the unwrapped phase: Geophysical Journal International, 197, no. 2, 975986. http://dx.doi.org/10.1093/gji/ggu025.

Duveneck, E., P. Milcik, P. M. Bakker, and C. Perkins, 2008, Acoustic VTI wave equations and their application for anisotropic reverse-time migration: $78^{\text {th }}$ Annual International Meeting, SEG, Expanded Abstracts, 2186-2190.

Gholami, Y., R. Brossier, S. Operto, A. Ribodetti, and J. Virieux, 2013, Which parameterization is suitable for acoustic vertical transverse isotropic full-waveform inversion? Part I: Sensitivity and trade-off analysis: Geophysics, 78, no. 2, R81-R105. http://dx.doi.org/10.1190/geo2012-0204.1.

Liu, Y., B. Chi, J. Yang, and L. Dong, 2014, A flexible method to calculate directions in waveform inversion: $84^{\text {th }}$ Annual International Meeting, SEG, Expanded Abstracts, http://dx.doi.org/10.1190/segam2014-0409.1.

Metivier, L., R. Brossier, S. Operto, and J. Virieux, 2014, Multiparameter FWI — An illustration of the hessian operator role for mitigating trade-off between parameter classes: Presented at the 6th Saint Petersburg International Conference \& Exhibition — Geosciences — Investing in the Future, EAGE.

Plessix, R.-E., and Q. Cao, 2011, A parametrization study for surface seismic full-waveform inversion in an acoustic vertical transversely isotropic medium: Geophysical Journal International, 185, no. 1, 539-556. http://dx.doi.org/10.1111/j.1365-246X.2011.04957.x.

Pratt, R., Z.-M. Song, P. Williamson, and M. Warner, 1996, Two-dimensional velocity models from wide-angle seismic data by wavefield inversion: Geophysical Journal International, 124, no. 2, 323-340. http://dx.doi.org/10.1111/j.1365-246X.1996.tb07023.x.

Shin, C., and D.-J. Min, 2006, Waveform inversion using a logarithmic wavefield: Geophysics, 71, no. 3, R31-R42. http://dx.doi.org/10.1190/1.2194523.

Tarantola, A., 1984, Inversion of seismic reflection data in the acoustic approximation: Geophysics, 49, 1259-1266. http://dx.doi.org/10.1190/1.1441754.

Virieux, J., and S. Operto, 2009, An overview of full-waveform inversion in exploration geophysics: Geophysics, 74, no. 6, WCC1-WCC26. http://dx.doi.org/10.1190/1.3238367. 
Woodward, M. J., 1992, Wave-equation tomography: Geophysics, 57, 1526. http://dx.doi.org/10.1190/1.1443179. 